\title{
NON-SIMPLE GEODESICS IN HYPERBOLIC 3-MANIFOLDS
}

\author{
KERRY N. JONES
}

ALAN W. REID *

\begin{abstract}
Chinburg and Reid have recently constructed examples of hyperbolic 3manifolds in which every closed geodesic is simple. These examples are constructed in a highly non-generic way and it is of interest to understand in the general case the geometry of and structure of the set of closed geodesics in hyperbolic 3-manifolds. For hyperbolic 3-manifolds which contain an immersed totally geodesic surfaces there are always non-simple closed geodesics. Here we construct examples of manifolds with non-simple closed geodesics and no totally geodesic surfaces.
\end{abstract}

\section{SECTION 0 - Introduction}

By a hyperbolic 3-manifold (resp. orbifold) we shall always mean a complete orientable hyperbolic 3-manifold (resp. orbifold) of finite volume. Let $M$ be a hyperbolic 3-manifold, a closed geodesic in $M$ is called simple if it has no selfintersections, and non-simple otherwise.

As was recently shown in Chinburg and Reid [3], there are infinitely many commensurability classes of closed hyperbolic 3-manifolds all of whose closed geodesics are simple. It would seem, at least at the intuitive level, that this should be the "generic case" for hyperbolic 3-manifolds. However the examples in [3] are arithmetic, and their construction arises by interpreting the existence of a non-simple geodesic in terms of a quaternion algebra that is naturally associated to these groups. These examples are far from being understood in terms of hyperbolic geometry.

On the other hand many hyperbolic 3-manifolds contain immersions of a totally geodesic surface, and therefore must contain non-simple closed geodesics, as any hyperbolic 2-manifold of finite volume contains such closed geodesics. The focus of this paper is manifolds "that arise between these two extremes." Our main result shows that there are infinitely many commensurability classes of closed hyperbolic 3 -manifolds that contain a non-simple closed geodesic, but have no immersed totally geodesic surface. Again the methods appeal to arithmetic hyperbolic 3-manifolds. This is not surprising, since at present the only methods known to show that a hyperbolic 3-manifold cannot contain an immersed totally geodesic surface are also arithmetic in nature, cf. Maclachlan and Reid [9] and Reid [11] and [13].

1991 Mathematics Subject Classification. Primary: 57M50, Secondary: 30F40.

Key words and phrases. hyperbolic 3-manifold, geodesic.

* Supported in part by NSF DMS-9108050 


\section{Section 1 - Preliminaries}

For convenience we recall some salient points about arithmetic Kleinian groups and quaternion algebras. We refer the reader to Borel [2] and Vignéras [15] for details.

1.1. Let $k$ be a field of characteristic different from 2 . The standard notation for a quaternion algebra over $\mathrm{k}$ is the following. Let $a$ and $b$ be non-zero elements of $k$. Then $\left(\frac{a, b}{k}\right)$ denotes the quaternion algebra over $k$ with basis $\{1, i, j, i j\}$ subject to $i^{2}=a, j^{2}=b$ and $i j=-j i$.

Now assume $k$ is a number field, i.e. a finite extension of $\mathbb{Q}$. By a place $\nu$ of $k$ we will mean one of the canonical absolute values of $k$ defined in Lang, [7, pp. 34-35]. The finite places of $k$ correspond bijectively to the prime ideals of the ring of integers $R_{k}$ of $k$. An infinite place of $k$ is either real, corresponding to an embedding of $k$ into $\mathbb{R}$, or complex, corresponding to a pair of distinct complex conjugate embeddings of $k$ into $\mathbb{C}$. We refer the reader to $[\mathbf{7}$, p. 36] for the definition of the completion $k_{\nu}$ of $k$ at a place $\nu$. When $\nu$ is an infinite place, $k_{\nu}$ is isomorphic to $\mathbb{R}$ or $\mathbb{C}$ depending on whether $\nu$ is real or complex.

In addition, when $\nu$ is a finite place, the residue class field $\mathbb{F}_{\nu}$ associated to $\nu$ is the field $R_{\nu} / \pi_{\nu} R_{\nu}$ where $R_{\nu}$ is the ring of $\nu$-adic integers in $k_{\nu}$ and $\pi_{\nu}$ a local uniformizing parameter for $\nu$, see [7] for details. The field $\mathbb{F}_{\nu}$ is isomorphic to $R_{k} / P_{\nu}$ where $P_{\nu}$ is the k-prime associated to $\nu$.

Let $B$ be a quaternion algebra over the number field $k$. The classification of quaternion algebras $B_{\nu}=B \otimes_{k} k_{\nu}$ over the fields $k_{\nu}$ is quite simple. If $\nu$ is complex then $B_{\nu}$ is isomorphic to $M\left(2, k_{\nu}\right)$ over $k_{\nu}$. Otherwise there is up to isomorphism over $k_{\nu}$ a unique quaternion division algebra over $k_{\nu}$, and $B_{\nu}$ is isomorphic over $k_{\nu}$ to either this division algebra or to $M\left(2, k_{\nu}\right)$.

Classification of quaternion algebras in general is slightly more complicated. Again, let $B$ be a quaternion algebra over the number field $k$. B is ramified at a place $\nu$ of $k$ if $B_{\nu}$ is a division algebra. Otherwise we say $B$ is unramified at $\nu$. The classification theorem for quaternion algebras over number fields (see [15, Chapter $3]$ ) implies that the set $\operatorname{Ram}(B)$ of places of $k$ which ramify $B$ is finite and of even cardinality (and contains no complex places). Conversely, suppose $S$ is a finite set of places of $k$ which has even cardinality and which contains no complex places. Then there is a quaternion algebra $B$ over $k$ with $\operatorname{Ram}(B)=S$, and this $B$ is unique up to isomorphism over $k$. By [15, Chapter 2], a place $\nu$ of $k$ is ramified in $B=\left(\frac{a, b}{k}\right)$ exactly when the norm form of $B$, i.e., the quadratic form $x^{2}-a y^{2}-b z^{2}+a b w^{2}$ does not represent zero over $k_{\nu}$. Furthermore, if $\nu$ is a place that ramifies $B$ with associated prime ideal $\mathcal{P}$ then $\mathcal{P}$ appears in the factorization of the fractional ideal generated by $a$ or $b$, or $\mathcal{P}$ divides 2 , see [15, Chapter 2] or Lam [8, Chapter VI]. The set of finite places which ramify $B$ is denoted $\operatorname{Ram}_{f}(B)$.

1.2. Arithmetic hyperbolic 3 -manifolds are obtained as follows (cf. $[\mathbf{2}]$ and $[\mathbf{1 5}$, Chapter 4]).

Let $k$ be a number field having exactly one complex place. Let $B$ be a quaternion algebra over $k$ which ramifies at all real places of $k$. Let $\mathcal{O}$ be an order of $B$ and let $\mathcal{O}^{1}$ the group of elements of reduced norm 1 in $\mathcal{O}$. Over an embedding $k \hookrightarrow \mathbb{C}$ inducing the complex place of $k$ one may choose an algebra embedding 
$\rho: B \hookrightarrow M(2, \mathbb{C})$ which restricts to an injection $\rho: \mathcal{O}^{1} \hookrightarrow \operatorname{SL}(2, \mathbb{C})$. Let $P$ : $\mathrm{SL}(2, \mathbb{C}) \rightarrow \operatorname{PSL}(2, \mathbb{C})$ be the natural projection. Then $P \rho\left(\mathcal{O}^{1}\right)$ is a Kleinian group of finite covolume. An arithmetic Kleinian group $\Gamma$ is a subgroup of $\operatorname{PSL}(2, \mathbb{C})$ commensurable with a group of the type $P \rho\left(\mathcal{O}^{1}\right)$. We say $\Gamma$ is derived from a quaternion algebra if $\Gamma$ is actually a subgroup of some $P \rho\left(\mathcal{O}^{1}\right)$. We call $Q=\mathbb{H}^{3} / \Gamma$ arithmetic or derived from a quaternion algebra if $\Gamma$ is arithmetic or derived from a quaternion algebra. The quotient $Q$ is compact if and only if the algebra $B$ is a division algebra, which occurs precisely when some place of $k$ is ramified in $B$, see [15].

It is shown in $[\mathbf{9}]$ that a Kleinian group of finite co-volume is arithmetic if and only if the group $\Gamma^{(2)}=g p\left\{\gamma^{2}: \gamma \in \Gamma\right\}$ is derived from a quaternion algebra.

1.3. As described in Neumann and Reid [10], to any Kleinian group $\Gamma$ of finite co-volume we can associate a quaternion algebra over a number field. Indeed as discussed in $[\mathbf{1 0}]$ one can associate a quaternion algebra over a number field which is an invariant of the commensurability class of $\Gamma$. The algebra is $\mathrm{A}^{(2)}$ (see below) defined over the field $\mathbb{Q}\left((\operatorname{tr} \gamma)^{2}: \gamma \in \Gamma\right)$. This field was shown to be an invariant of the commensurability class of $\Gamma$ in Reid [12]. In the arithmetic case this coincides with the algebra described above. Moreover the construction of this algebra has nothing to do with discreteness.

Let $\Gamma$ be a non-elementary subgroup of $\operatorname{SL}(2, \mathbb{C})$, not necessarily discrete, and let $k=\mathbb{Q}(\operatorname{tr}(\gamma): \gamma \in \Gamma)$ be the trace-field, which of course need not be a number field. Define the associated quaternion algebra

$$
\mathrm{A} \Gamma=\left\{\Sigma a_{i} \gamma_{i}: a_{i} \in k, \gamma_{i} \in \Gamma\right\}
$$

where all sums are finite. That this is a quaternion algebra over $k$ is shown in Bass [1] for instance. We are using a different notation from $[\mathbf{3}]$ and $[\mathbf{1 0}]$ where $\mathrm{A} \Gamma$ denoted the invariant quaternion algebra.

We now indicate how to reconstruct the quaternion algebra from any pair of non-commuting elements of $\Gamma$. The proof of the following Lemma is completely analogous to that of Proposition 2 of Takeuchi [14]; see also Hilden, Lozano and Montesinos [6].

\section{Lemma 1.1.}

Let $\Gamma$ be a non-elementary subgroup of $\operatorname{SL}(2, \mathbb{C})$, and let $\gamma$ and $\delta$ be a pair of non-commuting hyperbolic elements of $\Gamma$. Then,

$$
\mathrm{A} \Gamma \cong\left(\frac{\left(\operatorname{tr}^{2}(\gamma)-4\right),(\operatorname{tr}([\gamma, \delta])-2)}{k}\right)
$$

For our purposes we require the following result which is almost a tautology, but is of some interest as it gives a trivial method of proving discreteness.

\section{Lemma 1.2.}

Let $\Gamma$ be a non-elementary subgroup of $\mathrm{SL}(2, \mathbb{C})$ such that the traces of $\Gamma$ consist of algebraic integers, the trace field of $\Gamma$ is a number field with one complex place and $\mathrm{A} \Gamma$ is ramified at all real places. Then $\Gamma$ is discrete and may be projectivized and embedded in a Kleinian group of finite covolume. 
Proof. The assumption on the traces imply that $\Gamma$ is contained in the norm one elements of an order $\mathcal{O}$ of $\mathrm{A} \Gamma$, see $[\mathbf{1}]$. However the conditions on the trace-field and $\mathrm{A} \Gamma$ imply that $P\left(\mathcal{O}^{1}\right)$ is an arithmetic Kleinian group by $\S 1.2$. Hence $\Gamma$ is discrete.

1.4. To complete our discussion of arithmetic Kleinian groups, we recall one result of [11] and [13] concerning totally geodesic surfaces in arithmetic hyperbolic 3manifolds. In particular the following theorem is proved in $[\mathbf{1 1}]$ extending $[\mathbf{9}]$ (see also $[\mathbf{1 3}])$.

\section{Theorem 1.3.}

Let $M=\mathbb{H}^{3} / \Gamma$ be a closed arithmetic hyperbolic 3-manifold with invariant tracefield $k$ and invariant quaternion algebra $A$. $M$ contains an immersion of a totally geodesic surface if and only if the following conditions are satisfied:

(1) $[k: k \cap \mathbb{R}]=2$;

(2) $\operatorname{Ram}_{f}(A)=\emptyset$ or $\operatorname{Ram}_{f}(A)=\left\{\nu_{\mathcal{P}_{1}}, \nu_{\mathcal{P}_{1}^{\prime}}, \ldots, \nu_{\mathcal{P}_{r}}, \nu_{\mathcal{P}_{r}^{\prime}}\right\}$ where $\mathcal{P}_{i} \cap R_{k \cap \mathbb{R}}=$ $\mathcal{P}_{i}^{\prime} \cap R_{k \cap \mathbb{R}}=p_{i}$, for $i=1, \ldots, r$, where $p_{i}$ is a prime ideal of $k \cap \mathbb{R}$.

For convenience we state a corollary of this result that suffices for our needs.

\section{Corollary 1.4.}

Let $M=\mathbb{H}^{3} / \Gamma$ be an arithmetic hyperbolic 3-manifold such that the invariant quaternion algebra $\mathrm{A}$ is defined over $\mathbb{Q}(\sqrt{-d})$, where $d$ is a square-free positive integer. If $A$ is ramified at a place $\nu$ which lies over an inert place in $\mathbb{Q}$, then $M$ does not contain an immersed totally geodesic surface.

\section{Section 2 - Construction}

For the purpose of constructing these manifolds, we recall a few facts about traces in $\mathrm{SL}(2, \mathbb{C})$ and their relation to hyperbolic geometry. Note that we will be working in $\operatorname{SL}(2, \mathbb{C})$ rather than in $\operatorname{PSL}(2, \mathbb{C})$. When we say that an isometry of $\mathbb{H}^{3}$ is represented by a matrix in $\mathrm{SL}(2, \mathbb{C})$, we assume a fixed representation from $\operatorname{Isom}^{+}\left(\mathbb{H}^{3}\right)$ to $\operatorname{PSL}(2, \mathbb{C})$ and a fixed, consistent lifting of the image group to $\mathrm{SL}(2, \mathbb{C})$.

Proposition 2.1. Let $\alpha, \beta \in \mathrm{SL}(2, \mathbb{C})$. Then,

(1) $\operatorname{tr}(\alpha) \operatorname{tr}(\beta)=\operatorname{tr}(\alpha \beta)+\operatorname{tr}\left(\alpha \beta^{-1}\right)$

(2) $\operatorname{tr}([\alpha, \beta])=\operatorname{tr}^{2}(\alpha)+\operatorname{tr}^{2}(\beta)+\operatorname{tr}^{2}(\alpha \beta)-\operatorname{tr}(\alpha) \operatorname{tr}(\beta) \operatorname{tr}(\alpha \beta)-2$

(3) if $\alpha$ is diagonal and $\beta=\left(\begin{array}{ll}a & b \\ c & d\end{array}\right)$, then $\operatorname{tr}([\alpha, \beta])-2=-b c\left(\operatorname{tr}^{2}(\alpha)-4\right)$

Proof. Parts (1) and (2) are standard trace identities (valid over $\operatorname{SL}(2, R)$ for any commutative ring $\mathrm{R}$ with identity) so we omit the proof.

For part (3), we let $\alpha=\left(\begin{array}{cc}\lambda & 0 \\ 0 & \lambda^{-1}\end{array}\right)$ and simply calculate directly that

so that

$$
\operatorname{tr}([\alpha, \beta])=2 a d+\left(\lambda^{2}+\lambda^{-2}\right)(1-a d)
$$

$$
\begin{aligned}
\operatorname{tr}([\alpha, \beta])-2 & =2(a d-1)+\left(\lambda^{2}+\lambda^{-2}\right)(1-a d) \\
& =(1-a d)\left(\lambda^{2}-2+\lambda^{-2}\right) \\
& =-b c\left(\operatorname{tr}^{2}(\alpha)-4\right)
\end{aligned}
$$


Proposition 2.2. Let $x$ be an hyperbolic or elliptic isometry of $\mathbb{H}^{3}$ represented by $\alpha \in \mathrm{SL}(2, \mathbb{C})$. Let the translation distance of $x$ be $\rho$ and the rotation angle (torsion) be $\theta$. Then,

$$
\rho+i \theta=2 \cosh ^{-1}\left(\frac{\operatorname{tr}(\alpha)}{2}\right)
$$

Proof. Conjugate so that $\alpha$ is diagonal, and use the fact that $\cosh ^{-1}(z)=\ln (z+$ $\left.\sqrt{z^{2}-1}\right)$.

Proposition 2.3. Let $\alpha, \beta \in \mathrm{SL}(2, \mathbb{C})$ be matrices representing non-commuting hyperbolic or elliptic isometries $x, y$ (respectively) of $\mathbb{H}^{3}$ with the fixed points of $x$ and $y^{-1} x y$ all distinct. Let $z$ be the isometry with axis mutually perpendicular to the axis of $x$ and the axis of $y^{-1} x y$ taking the axis of $x$ to the axis of $y^{-1} x y$. Let $z$ be represented by a matrix $\gamma$. Then,

$$
\operatorname{tr}(\gamma)=2 \sqrt{1-\frac{\operatorname{tr}([\alpha, \beta])-2}{\operatorname{tr}^{2}(\alpha)-4}}
$$

Proof. First, conjugate $\alpha$ and $\beta$ into the form

$$
\alpha=\left(\begin{array}{cc}
\lambda & 0 \\
0 & \lambda^{-1}
\end{array}\right), \quad \beta=\left(\begin{array}{cc}
a & b \\
c & d
\end{array}\right), \quad(a d-b c=1)
$$

and note that the condition on the fixed points forces $a, b, c, d$ to be nonzero. Next, conjugate by an isometry $\varphi=\left(\begin{array}{ll}p & q \\ s & t\end{array}\right)$ which takes the fixed points of $\alpha$ to 1 and -1 and takes the fixed points of $\beta^{-1} \alpha \beta$ to $\omega$ and $-\omega$ for some $\omega$ in $\mathbb{C}$. This requires that $(p z+q) /(s z+t)$ take $(0, \infty, b / d, a / c)$ to $(1,-1, \omega,-\omega)$. The first two constraints force $p=-s$ and $q=t$. Thus, $\varphi=\left(\begin{array}{cc}p & q \\ -p & q\end{array}\right)$. The remaining constraint is that

$$
\omega=\frac{p \frac{b}{d}+q}{-p \frac{b}{d}+q}=-\frac{p \frac{a}{c}+q}{-p \frac{a}{c}+q}
$$

or, $p^{2} a b=q^{2} c d$. Without loss of generality, take $q=1, p= \pm \sqrt{\frac{c d}{a b}}$ ( $\varphi$ may not be in $\operatorname{SL}(2, \mathbb{C})$ with this choice, but it doesn't matter, since we are only conjugating by $\varphi$ ). Then,

$$
\omega=\frac{\sqrt{a d} \pm \sqrt{b c}}{\sqrt{a d} \mp \sqrt{b c}}=a d+b c \pm 2 \sqrt{a d b c}
$$

since $a d-b c=1$. Note that $\omega^{-1}=a d+b c \mp 2 \sqrt{a d b c}$.

At this point, we see that $z$ is realized by the matrix $\left(\begin{array}{cc}\sqrt{\omega} & 0 \\ 0 & \sqrt{\omega^{-1}}\end{array}\right)$. Thus, $\operatorname{tr}(\gamma)=\sqrt{\omega}+\sqrt{\omega^{-1}}$. We need to calculate this in terms of the traces of the original $\alpha$ and $\beta$. Using Proposition 2.1(1), we see that

$$
\operatorname{tr}^{2}(\gamma)=\omega+\omega^{-1}+2=2 a d+2 b c+2=4(1+b c)
$$

Proposition 2.1(3) yields $\operatorname{tr}([\alpha, \beta])-2=-b c\left(\operatorname{tr}^{2}(\alpha)-4\right)$ so that

$$
\operatorname{tr}(\gamma)=2 \sqrt{1-\frac{\operatorname{tr}([\alpha, \beta])-2}{\operatorname{tr}^{2}(\alpha)-4}}
$$

as claimed. 
Proposition 2.4. Let $z_{1}(\neq \pm 2), z_{2}, z_{3} \in \mathbb{C}$. Then, there exist $\alpha, \beta \in \mathrm{SL}(2, \mathbb{C})$ such that $\operatorname{tr}(\alpha)=z_{1}, \operatorname{tr}(\beta)=z_{2}$, and $\operatorname{tr}([\alpha, \beta])=z_{3}$.

Proof. Let

$$
\alpha=\left(\begin{array}{cc}
a & 0 \\
0 & a^{-1}
\end{array}\right), \quad \beta=\left(\begin{array}{cc}
b & b c-1 \\
1 & c
\end{array}\right)
$$

Then, Proposition 2.1(3) yields

$$
\frac{\operatorname{tr}([\alpha, \beta])-2}{\operatorname{tr}^{2}(\alpha)-4}=1-b c
$$

So, to satisfy the conclusion of the Proposition, we need merely find $a, b, c$ such that

(1) $a+a^{-1}=z_{1}$

(2) $b+c=z_{2}$

(3) $1-b c=\left(z_{3}-2\right) /\left(z_{1}^{2}-4\right)$

These are solved by setting $a=\left(z_{1} \pm \sqrt{z_{1}^{2}-4}\right) / 2, \lambda=\left(z_{3}-2\right) /\left(z_{1}^{2}-4\right), b=$ $\left(z_{2} \pm \sqrt{z_{2}^{2}-4+4 \lambda}\right) / 2, c=z_{2}-b$.

Putting all these pieces together, we have the following. We point out that by closed geodesic in a hyperbolic 3-orbifold, we mean: the image of a circle in the orbifold that lifts to a geodesic in $\mathbb{H}^{3}$. The geodesic is simple if the distinct geodesics in the lift do not intersect. Otherwise the closed geodesic is non-simple.

Theorem 2.5. Let $p, q, d \in \mathbb{Z}^{+}$with $d$ prime, and $p<q$. Let $a, b \neq \pm 2$ be algebraic integers in $\mathbb{Q}(\sqrt{-d})$ such that there exist algebraic integers $x, y$ in $\mathbb{Q}(\sqrt{-d})$ with

(1) $a^{2}-4=q x$

(2) $q x\left(b^{2}-4\right)+4 p x=y^{2}$

Then, there is a Kleinian group $\Gamma$ of finite covolume such that $\Gamma$ contains elements of trace $a$ and trace $b$ and the orbifold $\mathbb{H}^{3} / \Gamma$ contains a non-simple closed geodesic.

Proof. We will apply Proposition 2.4 with $z_{1}=a, z_{2}=b$ and $z_{3}=p x+2$ to obtain matrices $\alpha, \beta \in \mathrm{SL}(2, \mathbb{C})$ with $\operatorname{tr}(\alpha)=a, \operatorname{tr}(\beta)=b$, and $\operatorname{tr}([\alpha, \beta])=p x+2$. Applying Proposition 2.3 to $\alpha$ and $\beta$, we find that the isometry taking the axis of $\alpha$ to the axis of $\beta^{-1} \alpha \beta$ and having axis mutually perpendicular to these two axes is elliptic, since its trace is $2 \sqrt{1-p / q}$. This is equivalent to showing that the axes of $\alpha$ and $\beta^{-1} \alpha \beta$ intersect. In fact, using Proposition 2.2, we see that $p / q=\cos ^{2}(\theta / 2)$ where $\theta$ is the intersection angle of the two axes. Thus, we have our non-simple geodesic, provided we have a Kleinian group. We need only to show now that $\langle\alpha, \beta\rangle$ may be embedded in a finite covolume Kleinian group.

Note that if $\operatorname{tr}(\alpha), \operatorname{tr}(\beta)$ and $\operatorname{tr}([\alpha, \beta])$ are all algebraic integers, then $\operatorname{tr}(\alpha \beta)$ must be an algebraic integer, since, by Proposition 2.1(2) it satisfies a monic polynomial with algebraic integer coefficients. By assumption, $\operatorname{tr}(\alpha)$ and $\operatorname{tr}(\beta)$ lie in $O_{d}$, the ring of integers in $\mathbb{Q}(\sqrt{-d})$. Thus using Lemma 1.2 to construct $\Gamma$ it suffices to show that $\operatorname{tr}(\alpha \beta)$ also lies in $O_{d}$, for then every trace in $\langle\alpha, \beta\rangle$ is an integer combination of these traces, and as we are dealing with algebras over $\mathbb{Q}(\sqrt{-d})$, every quaternion algebra over this field gives rise to arithmetic Kleinian groups, possibly non-cocompact. 
The assertion will follow, if the discriminant of the quadratic polynomial satisfied by $\operatorname{tr}(\alpha \beta)$ in 2.1(2) is a square in $\mathbb{Q}(\sqrt{-d})$. This follows from the calculation below.

$$
\begin{aligned}
-4\left(\operatorname{tr}^{2}(\alpha)+\operatorname{tr}^{2}(\beta)-\operatorname{tr}([\alpha, \beta])-2\right) & \\
+\operatorname{tr}^{2}(\alpha) \operatorname{tr}^{2}(\beta)= & \left(\operatorname{tr}^{2}(\alpha)-4\right)\left(\operatorname{tr}^{2}(\beta)-4\right) \\
& +4(\operatorname{tr}([\alpha, \beta])-2) \\
= & q x\left(b^{2}-4\right)+4 p x \\
= & y^{2}
\end{aligned}
$$

In Theorem 2.5, the algebra $\mathrm{A} \Gamma$ may be the matrix algebra. However, we can arrange that this will not be the case as we now proceed to show.

We compute the Hilbert symbol of A $\Gamma$, using Lemma 1.1 and the following two basic facts about Hilbert symbols. First, the entries of a Hilbert symbol may be freely multiplied by nonzero squares in $K$ without altering the isomorphism class of the quaternion algebra. Second,

$$
\left(\frac{a, b}{K}\right) \sim\left(\frac{-a b, a}{K}\right)
$$

where $\sim$ denotes the relation of having isomorphic algebras. Thus, we compute that the Hilbert symbol of $\mathrm{A} \Gamma$ is

$$
\left(\frac{p x, q x}{\mathbb{Q}(\sqrt{-d})}\right) \sim\left(\frac{-p q x^{2}, q x}{\mathbb{Q}(\sqrt{-d})}\right) \sim\left(\frac{-p q, a^{2}-4}{\mathbb{Q}(\sqrt{-d})}\right)
$$

So, whenever there is a prime ramifying this algebra, we will have $\mathbb{H}^{3} / \Gamma$ compact.

More important for our purposes here is the following corollary of our discussions above.

Corollary 2.6. Given any positive prime $d$ and $m$ an algebraic integer in $\mathbb{Q}(\sqrt{-d})$, there is a Kleinian group $\Gamma$ of finite covolume such that the Hilbert symbol of $\mathrm{A} \Gamma$ is

$$
\left(\frac{-3, m^{2}-1}{\mathbb{Q}(\sqrt{-d})}\right)
$$

and the orbifold $\mathbb{H}^{3} / \Gamma$ contains a non-simple closed geodesic.

Proof. Apply Theorem 2.5 with $p=3, q=4, a=2 m, b=m$. Then, $x=m^{2}-1$ and

$$
\begin{aligned}
q x\left(b^{2}-4\right)+4 p x & =4\left(m^{2}-1\right)\left(m^{2}-4\right)+12\left(m^{2}-1\right) \\
& =4\left(m^{2}-1\right)\left(m^{2}-4+3\right) \\
& =4\left(m^{2}-1\right)^{2}
\end{aligned}
$$

so we may take $y=2\left(m^{2}-1\right)$. Since squares may be freely removed from the entries in the Hilbert symbol, we remove factors of 4 from both entries of the Hilbert symbol calculated following the proof of Theorem 2.5 to obtain the asserted Hilbert symbol. 
We note that none of the groups constructed here are torsion-free: $\operatorname{tr}(\alpha \beta)=1$ so $\alpha \beta$ is elliptic of order 3. We need one further result to be able to construct manifolds with a non-simple geodesic. We will do this by constructing an appropriate torsionfree subgroup of finite index. That is, we produce a torsion-free subgroup of finite index in the group $\Gamma$ above containing $\beta$. For then it will also contain some power of $\alpha$, and thus will still contain a non-simple closed geodesic. Most techniques for constructing torsion-free, finite index subgroups in Kleinian groups do not allow one to include an arbitrary non-torsion element in the subgroup. To do this, we need the following result which is of interest in its own right. For it seems that it is unknown in general that, given a hyperbolic 3-orbifold group $\Gamma$ and $\gamma \in \Gamma$ a loxodromic element, there is a torsion-free finite index subgroup that contains $\gamma$.

Remark. We have been informed by Peter Scott that this is true for Fuchsian groups, and the method of proof uses strong separability properties of such groups which are not known to be true for hyperbolic 3-orbifold groups.

Theorem 2.7. Let $\Gamma$ be a Kleinian group whose traces consist of algebraic integers. Let $k$ denote the trace-field of $\Gamma$. Let $a_{1}, a_{2}, \ldots, a_{n}$ be a finite subset of $R_{k}$ and let $\gamma \in \Gamma$. Suppose there exists a prime ideal $p$ of $R_{k}$ such that

(1) $\operatorname{tr} \gamma-2 \in p$

(2) $a_{i}-2 \notin p$ for all $i=1,2, \ldots, n$

(3) $\nu_{p}$ does not ramify $\mathrm{A} \Gamma$.

Then, there exists a finite index subgroup $\Gamma^{\prime} \leq \Gamma$ such that $\gamma \in \Gamma^{\prime}$ and $\Gamma^{\prime}$ contains no elements of trace $a_{i}$ for any $i=1,2, \ldots, n$.

Proof. Since $\nu_{p}$ does not ramify A $\Gamma$, we have a homomorphism from A $\Gamma$ to $\mathrm{A} \Gamma \otimes_{k}$ $k_{\nu_{p}}$ and an isomorphism from there to $M\left(2, k_{\nu_{p}}\right)$. This, together with the inclusion of $\Gamma$ into $\mathrm{A} \Gamma$ gives a representation from $\Gamma$ into $\mathrm{SL}\left(2, k_{\nu_{p}}\right)$.

In fact, since the traces of elements of $\Gamma$ are algebraic integers, this representation may be chosen to have image in $\operatorname{SL}\left(2, \mathcal{O}_{\nu_{p}}\right)$ where $\mathcal{O}_{\nu_{p}}$ is the $p$-adic integers in $k_{\nu_{p}}$. Now, if we let $\pi$ be a local uniformizing parameter for $\mathcal{O}_{\nu_{p}}$, we get a representation $\varphi: \Gamma \rightarrow \mathrm{SL}(2, \mathbb{F})$ where $\mathbb{F}=\mathcal{O}_{\nu_{p}} / \pi \mathcal{O}_{\nu_{p}}$ is the residue class field at $p$ (recall $\S 1$ ).

Since $\operatorname{tr} \gamma-2 \in p, \varphi(\gamma)$ is the identity or is unipotent. In either case, $\operatorname{tr} \varphi\left(\gamma^{m}\right)=2$ for all $m$. Let $\Gamma^{\prime}=\varphi^{-1}(\langle\varphi(\gamma)\rangle)$. Clearly, $\Gamma^{\prime}$ has finite index in $\Gamma$. Furthermore, no element in $\Gamma$ of trace $a_{i}$ is in $\Gamma^{\prime}$ since $a_{i}-2 \notin p$ by assumption.

We can now proceed to construct closed hyperbolic manifolds containing a nonsimple closed geodesic.

Theorem 2.8. Let $d$ be a prime congruent to 1 modulo 3. Let $n$ be an arbitrary algebraic integer in $\mathbb{Q}(\sqrt{-d})$. If we let $m=1+4 \sqrt{-d}+6 n$, then the Kleinian group $\Gamma$ constructed in Corollary 2.6 has a torsion-free finite index subgroup $\Gamma^{\prime}$ such that $\mathbb{H}^{3} / \Gamma^{\prime}$

(1) is a closed hyperbolic manifold

(2) contains no immersed totally geodesic surfaces

(3) contains a non-simple closed geodesic

Proof. First, observe that the only torsion elements of $\Gamma$ have order 2 or 3 , since the trace of an element of order $q$ is $\pm 2 \cos \pi / q$, and this by assumption must lie in $\mathbb{Q}(\sqrt{-d})$. 
We shall apply Theorem 2.7 to $\Gamma$ with $\gamma=\beta$ and $a_{1}=0, a_{2}=-1, a_{3}=+1$. First we observe that $m-2$ is not \pm 1 as $m$ is not real. As $d$ is congruent to $1(\bmod 3), \pm 1$ are the only units, hence there is a non-trivial prime ideal $\mathcal{P}$ of $\mathbb{Q}(\sqrt{-d})$ containing $m-2$.

Now $\mathcal{P}$ cannot contain $-3,-2$, or -1 : clearly, $\mathcal{P}$ cannot contain -1 . If $\mathcal{P}$ contains -2 then $\mathcal{P}$ contains $m-4 \sqrt{-d}-6 n=1$ which is impossible. Finally since $d$ is congruent to $1(\bmod 3), 3$ does not divide the norm of $m-2=-1+4 \sqrt{-d}+6 n$.

As discussed in $\S 1.1$ for any place ramifying the algebra $\mathrm{A} \Gamma$ of Corollary 2.6, the associated prime ideal divides $\langle 3\rangle,\left\langle m^{2}-1\right\rangle$ or $\langle 2\rangle$. Thus the ideal $\mathcal{P}$ above cannot ramify the algebra, since it would then necessarily contain either $2,-3, m-1$, or $m+1$. Thus, Theorem 2.7 applies to obtain a finite index, torsion-free $\Gamma^{\prime}$ with $\beta \in \Gamma^{\prime}$ and thus, we have part (3).

To prove (1) we shall show that the place $\nu$ of $\mathbb{Q}(\sqrt{-d})$ associated to the prime ideal $\langle 3\rangle$ (which is inert since $d \equiv 1(\bmod 3))$ ramifies the algebra $\mathrm{A} \Gamma$. For then as noted in $\S 1.2$, this ensures cocompactness of any group in the commensurability class determined by $\Gamma$. Moreover by Corollary 1.4, this also guarantees no totally geodesic surfaces.

To show that $\nu$ ramifies this algebra, from the discussion in $\S 1.1$ we are required to prove that the form $x^{2}+3 y^{2}-\left(m^{2}-1\right) z^{2}-3\left(m^{2}-1\right) w^{2}$ does not represent 0 over $\mathbb{Q}(\sqrt{-d})_{\nu}$. We have already observed above that $m^{2}-1$ is a $\nu$-adic unit, i.e., $\langle 3\rangle$ does not divide $\left\langle m^{2}-1\right\rangle$. By [8, Chap. VI, Proposition 1.9], to prove that $\nu$ ramifies it suffices to show that $x^{2}-\left(m^{2}-1\right) y^{2}$ does not represent zero over the residue class field $\mathbb{F}_{\nu}$, which is a finite field of order 9 . Since -1 is not a square modulo $3, \mathbb{F}_{\nu}=\mathbb{F}_{3}[\sqrt{-1}]$, where $\mathbb{F}_{3}$ is the finite field with 3 elements.

If this form did represent 0 , this would imply that $\left(m^{2}-1\right)$ is a square in $\mathbb{F}_{3}[\sqrt{-1}]$. However, as is easily checked, the only squares in this finite field are $\{0, \pm 1, \pm \sqrt{-1}\}$, and $m$ was chosen so that $m^{2}-1 \equiv-1-\sqrt{-1}(\bmod \nu)$. In particular $m^{2}-1$ is not a square and this completes the proof.

To get infinitely many commensurability classes we simply note that by Dirichlet's Theorem on primes in an arithmetic progression, there are infinitely many primes congruent to $1(\bmod 3)$.

\section{SeCtion 3 - Examples}

To construct simple examples of these manifolds, we first observe that the simplest trace-field to which our results apply is $\mathbb{Q}(\sqrt{-1})$ (although, strictly speaking, 1 isn't prime, we really only need $d$ to be non-composite). The algebraic integers in $\mathbb{Q}(\sqrt{-1})$ are the Gaussian integers $\mathbb{Z}[\sqrt{-1}]$. So, to find a simple example of a hyperbolic manifold of the type we have constructed, we seek a Gaussian integer $n$ such that $m=1+4 i+6 n$ generates a "fairly simple algebra" as in Corollary 2.6. By this we mean construct an algebra so that one obtains examples of small volume. It is well-known (cf. [2]) that the co-volume of a group $P \rho\left(\mathcal{O}^{1}\right)$, for $\mathcal{O}$ a maximal order, is related to the primes that ramify the algebra. On setting $n=-i$, we obtain $m=1-2 i$, so that one particular algebra is (after some simplification)

$$
\left(\frac{3,1+i}{\mathbb{Q}(i)}\right)
$$

This algebra is ramified at the places corresponding to the primes 3 and $1+i$ 
(the prime above 2). It can be checked that there is a unique type of maximal order (see $[\mathbf{1 5}]$ ), and that the co-volume of $P \rho\left(\mathcal{O}^{1}\right)$ is approximately $2.4425749 \ldots$

The Kleinian group corresponding to the elements of norm one in a maximal order of this algebra is an orbifold whose underlying space is the Lens Space $L(5,2)$ and having singular set a knot with isotropy group $\mathbb{Z}_{3}$. Since the singular set is a knot, there is a unique (up to conjugacy) maximal torsion-free subgroup (the fundamental group of the 3 -fold cyclic branched cover of $L(5,2)$, branched over the singular set of the orbifold). Now, this torsion-free subgroup will not necessarily be the torsion-free subgroup whose existence is guaranteed by Theorem 2.7, but some conjugate of it will contain that subgroup, and hence, the maximal torsionfree subgroup will also correspond to a closed hyperbolic manifold containing a non-simple geodesic and no immersed totally geodesic surfaces.

This orbifold is obtained by $(5,-2),(3,0)$ surgery on the $(3,-4,-4)$ pretzel link, where the knotted component has the $(3,0)$ surgery (see Fig. 3.1). Jeff Weeks' computer program, Snappea confirms that the volume of this orbifold is 2.4425749 . . .

The best presentation that we have found for this orbifold group has three generators and abelianization $\mathbb{Z}_{15}$. Two views of the fundamental domain for this orbifold (in the upper half-space model of hyperbolic space) are given in Fig. 3.2. Note that the face pairings are indicated on one of the views (looking down from above). Although we have not indicated the precise nature of the pairings by edge identifications, this is fairly clear because of the conformality of the upper half-space model. In the first view, the observer is situated slightly below the polyhedron and is looking at the face labelled "J" in the second view.

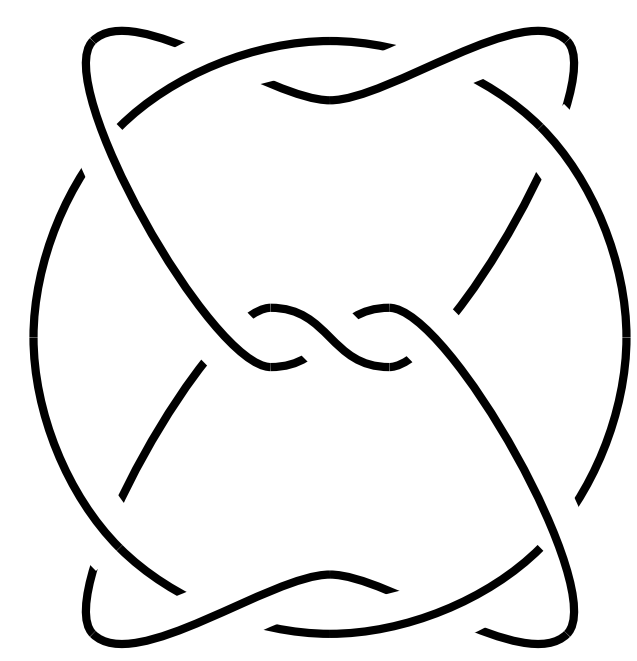

Figure 3.1

The maximal torsion-free subgroup (fundamental group of the manifold containing the non-simple geodesic) is a two-generator group and has first homology group $\mathbb{Z}_{8} \oplus \mathbb{Z}_{40}$. We should note, however, that this manifold has a double-cover with first homology $\mathbb{Z} \oplus \mathbb{Z}_{4} \oplus \mathbb{Z}_{20}$ and so the manifold is Haken, by a result of Hass [5], So, although it contains no (immersed) totally geodesic surfaces, it does nevertheless contain an (embedded) incompressible surface. This is in itself interesting, since the manifold has no totally geodesic surfaces, and at present the only other way of trying to show that an arithmetic hyperbolic 3-manifold has a finite cover which 
is Haken, is to use the results of Clozel [4]. However, these do not apply to this example, nor indeed to any of the examples given in the construction as 3 is inert.

A few words of explanation are in order as to how this example was computed. We have written computer programs which take as input an order in a quaternion algebra and calculate a fundamental domain for the Kleinian group consisting of the elements of norm one in the order (as well as other useful quaternion algebra calculations). More investigations using these computer programs will be discussed in a future paper. The great benefit of arithmetic Kleinian groups here is that most of the calculations may be carried out in integer arithmetic (by working relative to an integral basis for an order) and that the group elements may be enumerated with a fixed amount of memory (by enumerating the order elements, which form a lattice). At present, the program is limited to quadratic trace fields, but we plan to remove this limitation shortly.

The fundamental domain in Fig. 3.2 was obtained by the following process: First, find an order containing the group generated by $\alpha$ and $\beta$ as in the proof of Theorem 2.5 (the axis of $\alpha$ intersects its image under $\beta$, giving rise to the nonsimple closed geodesic). For this, we may take the order consisting of Gaussian integer linear combinations of the basis $\{I, \alpha, \beta, \alpha \beta\}$. Next, we find a maximal order containing this order. Maximal orders may be recognized in the following fashion: let $\left\{a_{1}, a_{2}, a_{3}, a_{4}\right\}$ be a basis for the order and let $A=\left(\operatorname{tr} a_{i} a_{j}\right)$ be the matrix of traces of products of these basis elements. Recall from [15, Chapter 1] the discriminant of such an order $d(\mathcal{O})$ is the integral ideal given by $d(\mathcal{O})^{2}=\langle\operatorname{det}(A)\rangle$. The order is maximal if and only if $d(\mathcal{O})$ is exactly the product of prime ideals for which the corresponding places ramify the quaternion algebra, see [15, Chapter 1].

A useful fact in our context is that if the basis is of the form $\{I, x, y, x y\}$ where $x$ and $y$ have determinant 1 , then $\langle\operatorname{det} A\rangle=\langle\operatorname{tr}[x, y]-2\rangle^{2}$. Using these two facts, it is straightforward to find a maximal order which will then contain some conjugate of the previous order.

In the case at hand, the maximal order is generated by $\{I, x, y, x y\}$ where $\operatorname{tr} x=$ $1+i, \operatorname{tr} y=1$ and $\operatorname{tr} x y=-1+2 i$. Then, we find the fundamental domain of the orbifold consisting of elements of norm 1 in this maximal order. We should note the computational importance of passing to a maximal order: in this example, the best fundamental domain for the original order that we were able to find has 130 faces, while the fundamental domain for the maximal order in Fig. 3.2 has 26 and is much more easily dealt with.

The assertion that this orbifold is obtained by $(5,-2),(3,0)$ surgery on the $(3,-4,-4)$ pretzel link is seen to be true by the following procedure: There are 3 edges in the fundamental domain that correspond to singular edges in the orbifold (the total cone angle around them is $2 \pi / 3$ ). We begin by "folding up" the fundamental domain along these edges, which all occur as edges between adjacent identified faces (labelled B,E and M in Fig. 3.2). We also keep track of the singular arcs so produced, which are now in the interior of the ball on whose boundary the remaining identifications are to be carried out. After doing this, we see that many of the faces are redundant. That is, there are regions consisting of multiple faces which are glued to each other as a unit. In fact, after amalgamating these regions into single faces, we are left with a single tetrahedron whose faces are identified in pairs to give $L(5,2)$, together with three singular arcs which are identified end-toend to give a knot in $L(5,2)$ (see Fig. 3.3). By isotoping the knot in $L(5,2)$, we may put it entirely inside a solid torus which is half of a Heegaard splitting for $L(5,2)$ 


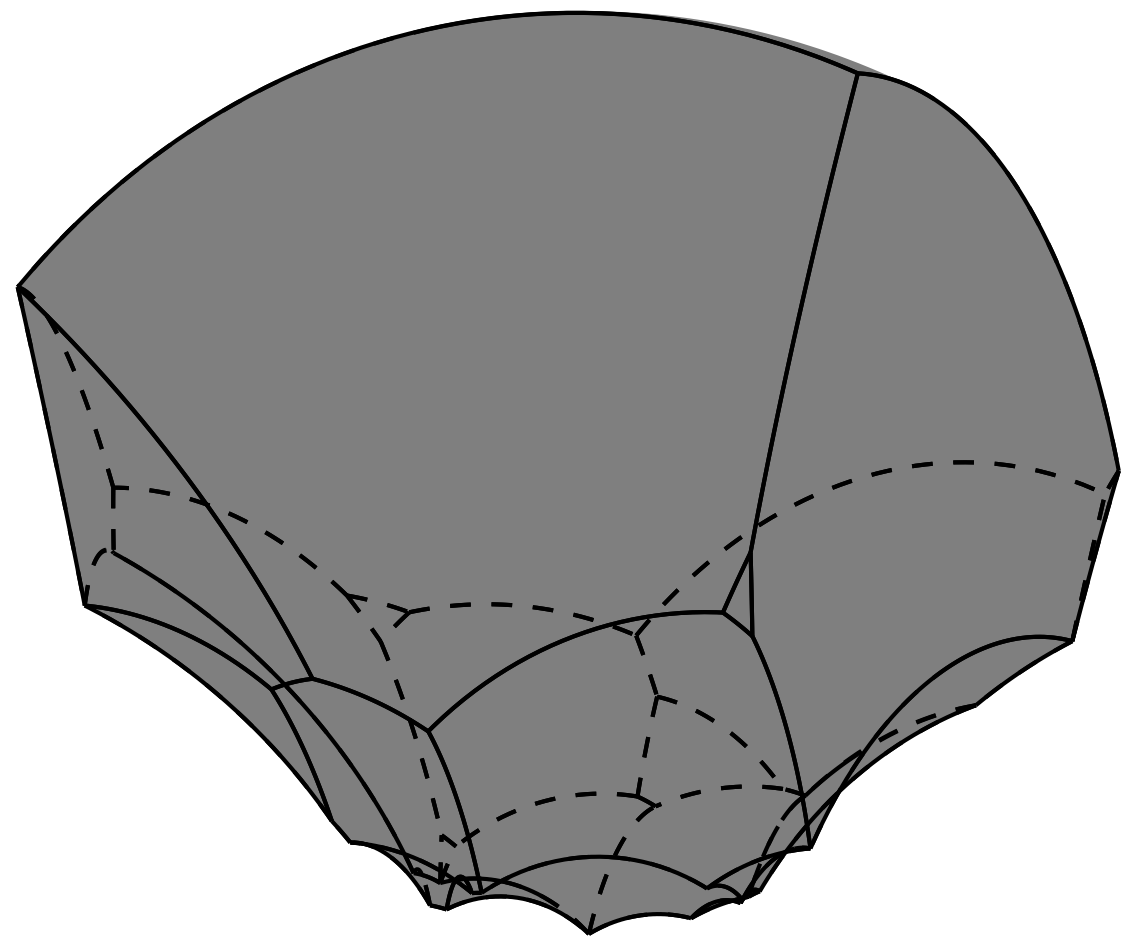

Figure 3.2(a)

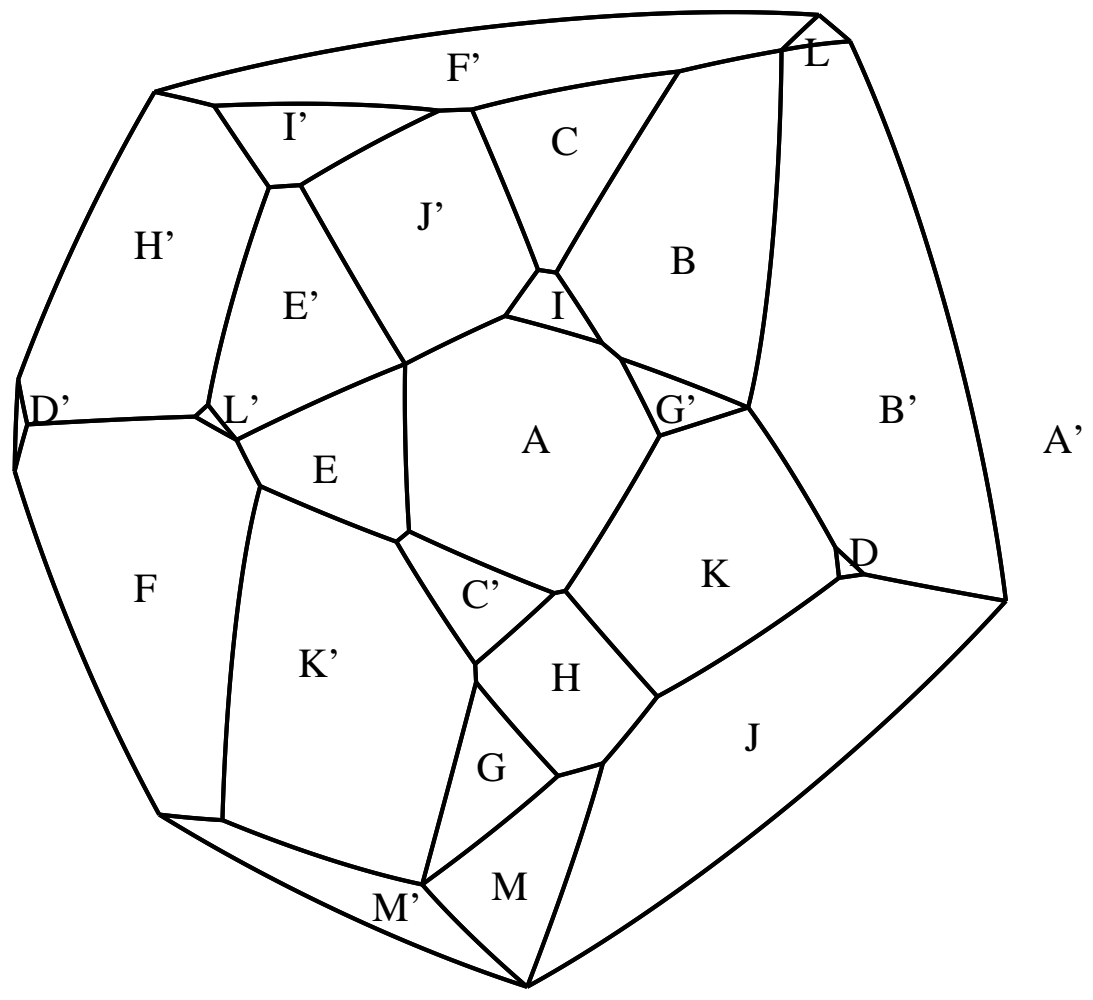

Figure 3.2(b) 

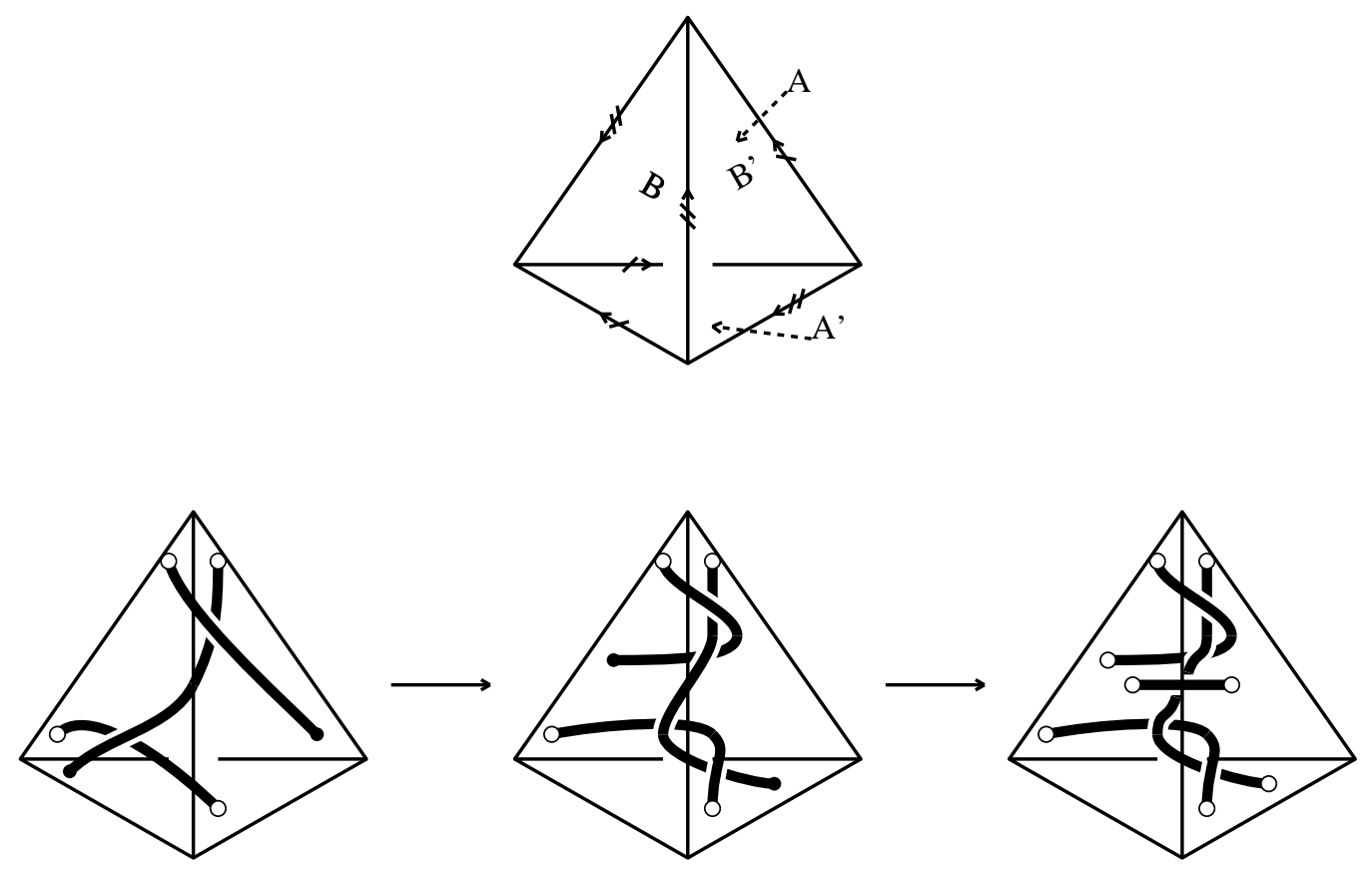

Figure 3.3

(the core of this solid torus is an unknotted arc between the centers of the two front faces in Fig. 3.3). We then embed this solid torus in $S^{3}$ to get a link complement on which the asserted surgery gives our orbifold. This link is readily seen to be the $(3,-4,-4)$ pretzel link. As a side note, the reader is invited to consider performing this procedure on the 130 -face fundamental domain corresponding to the original order!

\section{ACKNOWLEDGEMENTS}

The authors wish to thank the University of Texas at Austin for its hospitality and support while this research was being conducted.

\section{REFERENCES}

[1] H. Bass, Groups of integral representation type, Pacific J. Math. 86 (1980), 15-51.

[2] A. Borel, Commensurability classes and volumes of hyperbolic 3-manifolds, Ann. Scuola Norm. Sup. Pisa 8 (1981), 1-33.

[3] T. Chinburg and A. W. Reid, Closed Hyperbolic 3-manifolds all of whose Closed Geodesics are Simple, To appear J. Diff. Geom..

[4] L. Clozel, On the cuspidal cohomology of arithmetic subgroups of $S L(2 n)$ and the first betti number of arithmetic 3-manifolds, Duke Math. J. 55 (1987), 475-486.

[5] J. Hass, Surfaces minimizing area in their homology class and group actions on 3-manifolds, Math. Zeit. 199 (1988), 501-509.

[6] H. M. Hilden, M.-T. Lozano and J.-M. Montesinos, A characterization of arithmetic subgroups of $\mathrm{SL}(2, \mathbb{R})$ and $\mathrm{SL}(2, \mathbb{C})$, preprint.

[7] S. Lang, Algebraic Number Theory, Addison Wesley, U.S.A., 1970.

[8] T. Y. Lam, The Algebraic Theory of Quadratic Forms, Benjamin, U.S.A., 1973.

[9] C. Maclachlan and A. W. Reid, Commensurability classes of arithmetic Kleinian groups and their Fuchsian subgroups, Math. Proc. Camb. Phil. Soc. 102 (1987), 251-257.

[10] W. D. Neumann and A. W. Reid, Arithmetic of hyperbolic 3-manifolds, TOPOLOGY '90 (Proceedings of the Research Semester in Low Dimensional Topology, Ohio State University) 
(B. Apanasov, W.D. Neumann, A.W. Reid and L. Siebenmann, eds.), De Gruyter Verlag, Berlin, 1992, pp. 273-310.

[11] A. W. Reid, Arithmetic Kleinian groups and their Fuchsian subgroups, Ph.D. thesis, Univ. of Aberdeen, Aberdeen, 1987.

[12] _ _ A note on trace-fields of Kleinian groups, Bull. London Math. Soc. 22 (1990), 349352.

[13] (1991), 77-88.

[14] K. Takeuchi, Commensurability classes of arithmetic triangle groups, J. Fac. Sci. Univ. Tokyo Sec. IA 24 (1977), 201-212.

[15] M.-F. Vignéras, Arithmétique des algèbres de Quaternions, L.N.M. 800, Springer Verlag, Berlin, 1980.

Ball State University, Muncie, IN 47306 U.S.A.

E-mail address: $01 \mathrm{knjones@bsuvc.bsu.edu}$

UNIVERSITY OF CAMBRIDGE

E-mail address: A.W.Reid@pmms.cam.ac.uk 